\title{
Perceptual differences between reading handwritten and typed words
}

\author{
BONITA FORD \\ Claremont Graduate School, Claremont, California 91711 \\ and \\ WILLIAM P. BANKS \\ Pomona College, Claremont, California 91711
}

\begin{abstract}
Perceptual differences between reading handwritten and typed words were investigated in a series of three experiments. In the first two experiments, a Sternberg memory search paradigm was employed, with either typed or handwritten probes. The reaction time to classify handwritten probes was slower than for typed probes, but memory search took place at the same rate for both kinds of probe. In the third experiment, the latencies for naming the written and typed words differed by the same amount of time as the overall difference between the same stimuli in the memory search task. These findings indicate that only perceptual processes are differentially affected by the differences between typed and written words. Furthermore, in these tasks the independence of encoding and memory search makes it unlikely that processes of perceptual identification use expectations or analysis-by-synthesis based on the information in immediate memory.
\end{abstract}

It is a perhaps ironic fact that almost all studies of how humans read written words have used machine written (typed) words as stimuli, while most studies of how to make machines recognize words have used stimuli written by human hands (cf. Corcoran \& Rouse, 1970; Smith \& Spoehr, 1974; Uhr, 1963). The reasons for using different stimuli in the two cases are quite understandable, but the differences create important gaps in our knowledge of pattern recognition by man and machine. Largely because of the differences in materials, the results of psychological studies of word recognition are difficult to apply to the problem of machine reading of handwriting, and theories of perception derived from the machine perception literature have not predicted any interesting phenomena concerning human word perception. The most important distinction between written and typed words is not just the difference between the shapes of the letters in the two cases, but rather the fact that handwritten words vary in shape while typed words are constant. Thus, the paucity of studies of human perception of handwriting has meant that reading experiments have not generally studied one fundamental property of perception, namely, the way it copes so well with stimulus variability (cf. Kolers \& Perkins, 1975, for a discussion of this point in the context of reading studies).

This research was supported by Pomona College faculty research grants and by National Science Foundation Grant BMS 75-20328. Send requests for reprints to William $P$. Banks, Department of Psychology, Pomona College, Claremont, California 91711.
Corcoran and Rouse (1970) have examined perception of handwritten and typed words and have found evidence for what they term different "subroutines" for analyzing the two types of stimulus. They showed that recognition accuracy for either written or typed words is greater when they are presented in a blocked series of trials (i.e., long sequences of all typed or all handwritten words) than when they are randomly alternated from trial to trial. They interpreted their results to indicate that subjects can have just the typed or just the written "subroutine" ready to analyze the stimulus appropriately in the blocked condition, but cannot when the type of stimulus varies unpredictably from trial to trial. The subroutine for handwriting could not, of course, be just a template. It must include procedures for analyzing any type of handwriting that may be encountered, and thus be a fairly complex pattern recognizer.

The present experiments examine the question of whether the different subroutines for reading typing and handwriting hypothesized by Corcoran and Rouse (1970) are truly perceptual mechanisms of analysis, or whether they reflect strategies of inspection that depend on memory retrieval and "educated guesses." If the different subroutines are very early and automatic perceptual processes, they should be relatively independent of prior knowledge about the words or of memory-based perceptual strategies. The technique used in this study to distinguish memory and perception in reading words is the memory search paradigm of Sternberg $(1969,1975)$. While this paradigm is generally considered to be a method for analyzing short-term 
memory, it is perfectly suited for the present purpose.

In a typical Sternberg memory search task, the subject is presented a short list of items which he is to remember. He is then tested with a stimulus item (probe), his task being to respond with a "yes" if this item was in the list, and a "no" if it was not. The list that is committed to memory (memory set) may be varied from trial to trial (varied-set procedure) or may remain the same across large blocks of trials (fixed-set procedure). In his original experiment, Sternberg (1966) found that reaction time (RT) increases linearly with increases in memory set size (M).

Sternberg $(1969,1975)$ has presented evidence that supports at least four distinct processing stages in item recognition: (1) a stimulus encoding stage, where a representation of the probe is formed; (2) a serial comparison process in which the probe representation is compared in an exhaustive manner with items in the memory set; (3) a binary decision stage in which a decision is reached as to what type of response will be emitted; and (4) a response organization and execution stage. In the present experiments, we have focused only on the first two stages, being interested in whether the form of the probe will affect the stimulus encoding stage only, or whether it will have some effects on memory matching as well. Since mean RT is a linear function of memory set size, it is possible to separate the time used in the matching process from that used in the other stages, assuming the stages are additive. The slope of the RT function is taken to represent the amount of time required for each comparison of probe and memory set item. The zero intercept of the function is the measure of the mean time taken by all events other than the comparison process. If differences in the form of the probe affect the slope of the RT function, we may conclude that the matching stage has been affected. On the other hand, a change in intercept resulting from a change in stimulus characteristics would indicate that the stimulus encoding stage has been affected.

In the first two experiments reported here, the size of the memory set and the form of the probe (typed vs. handwritten) were varied orthogonally to determine whether differences between reading handwritten and typed words reflect strategies of examination that depend on memory. If perceptual analysis of the probes depended on an analysis-by-synthesis process (Neisser, 1967; cf. Kolers \& Perkins, 1975), for example, then probe identification should depend on the size of the memory set, assuming that memory set information would be retrieved for the "synthesis" part of analysisby-synthesis. The way to test for the use of memory would be to determine whether search functions for typed and handwritten probes differ in slope as well as intercept. If, on the other hand, perceptual differences exist at a very early stage of perceptual processing, prior to memory retrieval, then the two functions should differ only in intercept, having a constant RT difference at each memory set size.

The third experiment measured the naming latency for the same typed and written words that were used as probes in the memory search experiment. If the intercept difference found in the memory search experiments represents a difference in the duration of perceptual processing, then the mean naming latencies for the two kinds of stimuli should differ by the same absolute duration as the intercepts for the two search functions.

A second source of interest in the present research is in the use of the handwritten vs. typed variable as an analog of probe degradation. The existing experiments on the effects of probe degradation have generally shown that probe degradation affects only the encoding stage (Bracey, 1969; Checkosky \& Whitlock, 1973; Sternberg, 1967). However, these studies have used highly artificial techniques for degrading the probes. These include superimposing checkerboards and random dot patterns over the probes to degrade them. Not only do these forms of degradation have very low "ecological validity," but they are techniques that simply add irrelevant material that could, logically, be "subtracted" to reveal the probe. Thus, the possibility exists that some kind of relatively simple filtering strategy that has little to do with the normal processes of perception could be employed by subjects in the task. The observed independence between probe identification and memory search in these cases would then be found only because of the artificial nature and consistent form of the degradation. Handwritten words, on the other hand, differ from each other and from typed words in very complex ways. In this study, the probes were in the handwritings of many different individuals to increase the variability of the handwriting. No process of "filtering" that could "subtract" these differences seems possible. A finding of only an intercept difference between written and typed probes would serve as convincing evidence for a relatively sophisticated mechanism of pattern recognition that operates completely prior to processes that access information in active memory.

\section{EXPERIMENT 1}

\section{Method}

Subjects. The subjects in this experiment were eight female students from the Claremont Colleges. Subjects were paid for their participation in the experiment. In order to minimize errors, subjects had to pay 25 cents per error out of their total pay of $\$ 2.50$ per session.

Materials and apparatus. A Sternberg memory search paradigm was used, with memory sets of two, three, four, and five items. The stimuli used were words ranging from four to eight letters in length, taken from the Toronto Word Pool (Murdock \& Walker, 1969). Since the iterns in each memory set were chosen at random from the word pool, the memory sets consisted of words of different lengths. 
The typed probes were typed in uppercase using an IBM Selectric typewriter. The handwritten probes were written by 19 different people so that handwriting would be inconsistent from trial to trial. This was done so that subjects would not develop strategies specific to one person's handwriting. The 19 people each wrote about 20 different words, dictated by the experimenter, on the stimulus cards in the same location as the typed words. The written probes were drawn from this pool of about 380 words.

Stimuli were presented in an Iconix tachistoscope with approximately $20 \mathrm{~mL}$ of luminance in both stimulus and fixation fields and a viewing distance of $86 \mathrm{~cm}$. Both typed and handwritten words were seen near the center of the field and were lined up very closely with the fixation point. The visual angle of the probe varied from 1 to 2 deg (typed) and 1 to $4 \mathrm{deg}$ (written).

Design and procedure. Subjects participated in two test sessions held on consecutive days. Each session lasted approximately $45 \mathrm{~min}$. There was a rest period at midsession.

Memory sets of two, three. four, and five items were constructed, all using different words as stimuli. A given memory set was tested only once and no word was ever used in more than one memory set. The only words presented twice to the subjects were those that were positive probes. They appeared once in the memory set and once as probes. The three experimental variables (memory set size, typed vs. handwritten probe, and positive vs. negative response) were orthogonally combined and randomly presented from trial to trial, with the constraint that no value of any variable could occur more than three times in succession. There were a total of 16 cells in the experiment ( 4 by 2 by 2) and each was tested four times in a session for each subject, making 64 trials. In addition to the 64 experimental trials per session, practice sets were presented. The practice set for the first session consisted of 16 trials; that for the second session consisted of 6 .

Each trial began with the experimenter reading a new memory set. Items were read at the rate of approximately one per second. The last item was followed by an "OK" which indicated to the subject that she should squeeze the pedal and make a response. The subject made a verbal response that activated a voice key which stopped the timer and caused the fixation point to be substituted for the stimulus field. The RT was recorded and the next memory set read.

Subjects were informed of errors, and after an error was made an extra trial was inserted for which the RT was not recorded. At the end of the session, in order to obtain an equal number of reaction times for each cell, subjects were tested on all cc nditions in which they had made mistakes.

Subjects were randomly divided into two groups. These groups differed only in that the probes which were typed for one group were handwritten for the other and vice versa. In addition, each subject in one group had exactly the same randomly generated sequence of trials as one of the subjects in the other group. These matching procedures were used to insure that no systematic differences were confounded with the typed/written variable.

\section{Results}

Since the RTs for some conditions were negatively skewed, the median of the four RTs per condition was used as the representative score for each subject rather than the mean. These median RTs were subjected to an overall analysis of variance with subject, day, and response as between-blocks treatments, and probe form and memory set length as within-blocks treatments. All variables were treated as fixed, with the exception of the subject variable.

Figure 1 shows the means of the median RTs for

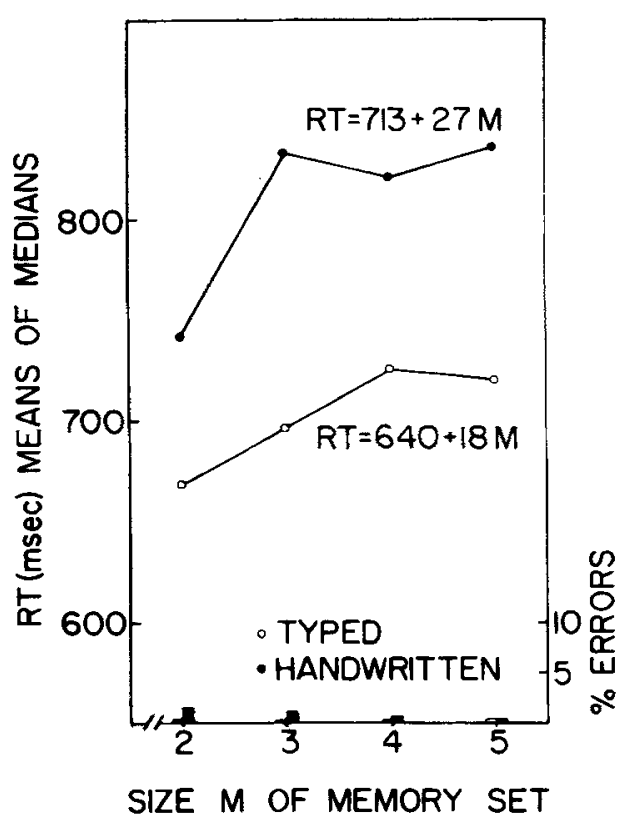

Figure 1. Means of median RTs and error percentage as functions of size of memory set for typed and handwritten probes for Experiment 1.

typed and handwritten probes plotted as a function of memory set. The median RTs for responses to both typed and handwritten probes increased with memory set size. There was a significant memory set effect $[F(3,31)=10.63, p<.01]$. The typed probes were processed faster than the written ones $[F(1,7)=16.93$, $\mathrm{p}<.01, \mathrm{MSe}=22.7 \mathrm{msec}]$. As can be seen in the figure, the written probe led to a search function about $50 \%$ steeper than the typed probe ( $18 \mathrm{vs.} 27 \mathrm{msec} / \mathrm{item})$. The interaction between set size and probe form was not reliable, however $[\mathrm{F}(3,21)=1.37]$. The small $\mathrm{F}$ ratio and the choppy nature of the functions suggest that the difference in slopes comes from error of measurement. Most of the difference in slope comes from the data for memory sets of two. The functions are roughly parallel for the range of three to five. Furthermore, the difference in slope comes entirely from the "no" responses. The memory search functions have the same slope for "yes" responses $(21 \mathrm{msec} /$ item for typed and $23 \mathrm{msec} /$ item for written probes), but they differ considerably for "no" responses $(15 \mathrm{msec} /$ item and $31 \mathrm{msec} / \mathrm{item}$ for typed and written probes, respectively). This difference between "yes" and "no" functions falls short of significance $[F(3,21)=2.30]$, but it is still large. The finding of an effect of probe type on "no" but not "yes" functions might be explained by a strategy difference in dealing with the two kinds of probes. When a subject did not find a match with the handwritten probe, she may have searched the list again, but not when the probe was typed and not when a match was made. The difference between slopes for handwritten and typed probes are thus possibly accounted for by differential subjective 
uncertainty rather than by an interaction between perception and memory. The second experiment will be seen to strengthen the conclusion that perception and memory do not interact in this task.

The analysis of the RTs showed a reliable difference between "yes" and "no" responses $[\mathrm{F}(1,7)=15.6$, $\mathrm{p}<.01]$ and a reliable three-way interaction among type of response, memory set size, and day $[F(3,21)=4.61$, $\mathrm{p}<.025]$. This triple interaction comes about because "yes" and "no" slopes were more nearly the same on the first day than the second. None of the other factors or their interactions approached significance. In particular, there was no reliable change in the pattern of slopes over the 2 days of testing.

Two separate analyses of variance were performed on the slopes and intercepts of the RT functions for each subject under each combination of conditions of probe type, response, and day. The analysis of slopes indicated that the effect of form of probe was not significant $[F(1,7)=3.0, M S e=3.88 \mathrm{msec} /$ item $]$, but the Day by Response interaction was significant $[F(1,7)=20.94, p<.01]$ (this interaction is equivalent to the linear component of the Response by Set Size by Day triple interaction reported above). The analysis of intercepts showed a reliable effect of form of probe $[F(1,7)=5.93, p<.05]$, intercepts for handwritten RT functions being higher than those for typed RT functions. The Day by Response interaction was significant $[F(1,7)=12.02, p<.025]$, as was the Day by Probe Form interaction $[F(1,7)=12.65$, $\mathrm{p}<.01]$. No other interactions were significant.

The results of this experiment indicate that only the probe encoding stage is interfered with when the probe is handwritten. The weak indication of an interaction between form of probe and memory set size was statistically unreliable, and because it held only for negative responses, it may have been the result of subjective strategies for dealing with uncertainty rather than an interaction between memory and perceptual processes. There were some methodological problems with this experiment, however. Subjects sometimes breathed heavily before making a response and activated the voice key prematurely. This may have been responsible for the skewed distributions of RTs obtained for subjects. The frequent triggering of the voice key by irrelevant sounds also contributed a good deal of error variability to the data and may have distorted the results in unknown ways. Because of these problems, we decided to replicate the experiment using a manual response rather than the voice key.

\section{EXPERIMENT 2}

\section{Method}

Subjects. The subjects for this experiment were eight female students from the Claremont Colleges. They received the same pay and instructions as those in Experiment 1.

Materials and apparatus. The materials used in this experiment were those used in Experiment 1. The only change introduced was in the apparatus. Instead of the voice key, two telegraph keys were used. The key for positive responses was on the subject's left, that for negative responses on her right. The subjects presented themselves the probe by stepping on a foot pedal when told to do so. They then made their response by tapping the appropriate key with their index finger.

\section{Results}

Figure 2 shows the mean RTs for typed and handwritten probes as a function of memory set. Mean RTs for responses to typed and handwritten probes increased at the same rate with memory set size. The effect of set size was significant $[F(3,21)=14.16$, $\mathrm{p}<.01]$, and the form of the probe had a highly significant main effect $[F(1,7)=35.86, p<.01]$, but type of response was not significant. None of the interactions was significant. The RTs were overall faster on the second day than on the first $[F(1,7)=11.26$, $\mathrm{p}<.025]$, but none of the interactions with day approached significance. The search functions for both days are quite smooth and look much like the plots in Figure 2, except for the overall displacement caused by the practice effect. The standard error of the mean for the slope difference was $3.86 \mathrm{msec} /$ item.

The analysis of variance for slopes yielded no significant effects. The analysis of intercepts indicated that the handwritten vs. typed variable had a significant effect $[F(1,7)=12.85, p<.01, \mathrm{MSe}=22.2 \mathrm{msec}]$. Type of response had no significant effect, and none of the interactions among any of the variables was significant.

The results of this experiment confirm those of Experiment 1 and indicate that only the perceptual

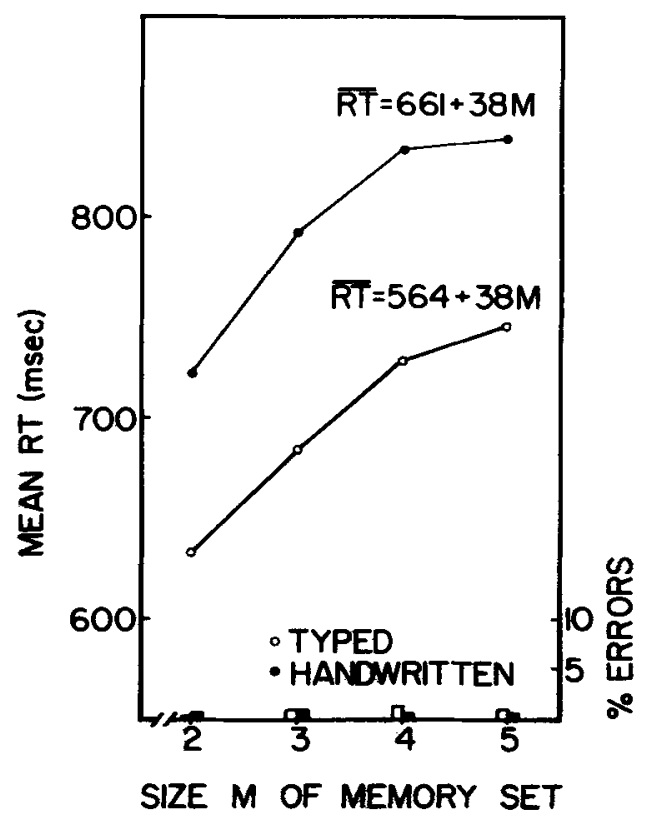

Figure 2. Mean RTs and error percentage as functions of size of memory set for typed and handwritten probes for Experiment 2. 
stage in the memory matching process is being interfered with by the handwriting. Experiment 3 tests to see if the form of the probe has an equivalent effect in a different task, namely, the reading aloud of singly presented probes.

\section{EXPERIMENT 3}

This experiment, performed concurrently with Experiment 1, measured the amount of time required to name the handwritten and typed probes used in the memory search task.

\begin{abstract}
Method
Subjects. The subjects in this experiment were those who participated in Experiment 1. This experiment was run on the second day of testing, upon completion of the memory search task.

Materials and apparatus. The materials used in this experiment were the probes which had been used on the first test day in Experiment 1, including those which had been in the practice list. Probes from the second test day were not used because there might have been some memory for probes and memory sets presented on that day, with a possible distortion of RTs to those probes. It was felt that memory for the previous day's probes would be negligible. The same apparatus was used in both experiments.

Design and procedure. The probes were shuffled after every two subjects and then presented one at a time in the tachistoscope. The subject's task was to name the word as quickly as possible. The timer started with the onset of the stimulus, and the subject's voice activated a voice $k e y$, which stopped the timer. When probes were identified incorrectly, the RT was not recorded.
\end{abstract}

\section{Results}

Median recognition times were computed for typed and handwritten probes for each subject. The mean of the medians for handwritten probes was $659.3 \mathrm{msec}$ and the mean of the medians for typed probes was $546.7 \mathrm{msec}[\mathrm{t}(7)=4.04, \mathrm{p}<.01]$. The difference of $112.6 \mathrm{msec}$ is approximately the same as the difference between the intercepts in the memory search task for the same subjects (Experiment 1).

Reaction time functions to typed and handwritten probes were computed for each subject from the data obtained during the first day of memory search. Data from the second day were not included, since the probes used on that day were not presented for naming. The intercept for the typed RT function was subtracted from that for the handwritten function for each subject. This measure represents the time taken up by probe clean-up in the perceptual stage of the memory matching process if the presence of handwriting affects only this stage and has no effect on the matching or other stages. The mean difference score was 108 msec.

To test whether the typed vs. handwriting variable affects only the perceptual processes, the intercept differences were compared with the recognition differences. The intercept differences were not significantly different from the recognition differences $[\mathrm{t}(7)=.18, \mathrm{MSe}=8.5 \mathrm{msec}]$.

\section{DISCUSSION}

In both Experiments 1 and 2, the intercepts for typed and handwritten functions differed reliably, whereas the slopes did not. The results indicate an independence of the search rate and probe form. None of the interactions was significant, indicating that the presence of handwriting contributed the same amount of processing time, regardless of memory set size or response, to the RT. It seems that only perceptual processes, and not memory comparison, are affected by the form of the probe. Further evidence for degradation affecting only the encoding stage was found in Experiment 3, where naming latency for the probes differed by about the same amount as the intercepts of the search functions for the same stimuli.

There are two possible sources of the mean overall difference in RT between typed and handwritten words. The first is the processing time required by the two perceptual "subroutines": The handwriting routine could simply take longer (about $100 \mathrm{msec}$ ) than the routine for typed letters to do its work, and thus cause the overall difference. The second is the switching time required to call up the proper routine if the correct one is not ready when the stimulus is presented. The mean difference in processing time between typed and handwritten words could come from any mixture of the two sources of a latency difference. There could, for example, be no difference at all in the time required by the two subroutines to operate, but a large difference in the subjects' preparedness to process the two kinds of stimuli. If the subjects always prepared themselves for typed probes, and if retrieving the writing subroutine on the trials when the probe was written took about $100 \mathrm{msec}$, then the observed mean difference between processing written and typed words would be predicted without any need for a difference between mean processing times for the two routines. On the other hand, if both routines were always simultaneously available and no time were ever required for switching, then the 100-msec difference in processing time would have to be attributed entirely to difference in the time the routines themselves take. Our data cannot be used to decide which factor causes the mean RT difference between handwritten and typed probes, but the data of Corcoran and Rouse (1970) suggest that both factors are involved to some extent. Accuracy of identification in their blocked condition, in which subjects can be assumed to have the correct routine ready on every trial, was greater than in their mixed condition, in which the correct subroutine varied unpredictably from trial to trial. However, there was an accuracy advantage for typed letters over written words in both conditions. Analysis of written letters, then, is still harder than analysis of typed letters, even when the appropriate perceptual subroutine is ready.

Whatever the reason may be for the slower processing of written than typed words, the fact that the difference 
was roughly constant over memory set size implies that the process of probe identification does not use the information contained in the memory set. If it did, the difficulty of accessing and using the information would increase with set size, memory load would add to the RT in both the encoding and memory search stages, and the RT for handwritten probes would necessarily increase more rapidly with memory set than the RT for typed probes. An equivalent way of stating this argument is in terms of Sternberg's (1967) analysis, according to which the parallel memory search functions indicate that the degraded (written) probe is converted to the same form as is the typed form by the time the memory comparison operations are begun. If the conversion process was not completed before memory comparison, it would have to take place at the time of each comparison and slow down the time per memory item, decreasing the rate of memory search. The fact that naming latency for the two kinds of probe differed by about the same amount as the intercepts strengthens the case against the use of information in short-term memory in the encoding process. The "memory set" for the naming task could be as large as the subject's reading vocabulary. It was certainly no smaller than the set of 64 probes used on the previous day, and it approaches that small size only under the unlikely assumption that memory for the previous day's probes is good.

What do these experiments tell us about the. perceptual process used to identify the probes? First, the nature of the variation in the form of the probewritten vs. typed-seems to preclude a simple and universally applicable "subtraction" routine that could automatically remove the difference between the difficult (written) and easy (typed) probes. Probe identification in this task seems, therefore, not to require information processing capacities that are specialized, arbitrary, or especially simple. They are probably as complex as those used in reading, and, in fact, the third experiment studies something very much like reading and finds the same estimate for the duration of the perceptual process as does the memory search task.

Second, the evidence seems very clear that this complex perceptual process operates independently of the information in active memory. The argument does not hinge on any model of memory search; the fact is that since the durations of the two processes are approximately additive in the RT, the two stages occupy separate, nonoverlapping blocks of time during processing. The perceptual process, therefore, does not seem to make use of the information in active memory to identify the stimulus. This nonuse of memory set information casts doubt on an analysis-by-synthesis model of word recognition (cf. Neisser, 1967). If analysis-by-synthesis (or some similar conceptually driven process) is used in the recognition of words, it either does not use the readily available hypotheses about the stimuli stored in the memory set or else it does not use them in anything like the manner in which they are interrogated in memory search. If perception did use the memory set items serially, the time required to use them would increase with memory set size and the search functions for handwritten and typed probes would not be parallel. The qualification that analysis-by-synthesis either does not use the memory set, or else uses it more efficiently and quickly than memory search itself does, makes the analysis-bysynthesis model implausible in this case. At this point, it therefore seems reasonable to assume that the perceptual mechanisms use only very general analytic routines and only the information available in the stimulus itself. The "context" provided by the memory set is apparently no help at all in the present task.

\section{REFERENCES}

BraCeY, G. W. Two operations in character recognition: A partial replication. Perception \& Psychophysics, 1969, 6, 357-360.

Checkosky, S. F., \& Whitlock, D. Effects of pattern goodness on recognition time in a memory search task. Journal of Experimental Psychology, 1973, 100, 341-348.

Corcoran, D. W. J., \& Rouse, R. O. An aspect of perceptual organization involved in reading typed and handwritten words. Quarterly Journal of Experimental Psychology, 1970, 22, 526-530.

Kolers, P. A., \& Perkins, D. N. Spatial and ordinal components of form perception and literacy. Cognitive Psychology, 1975, 7, 228-267.

Murdock, B. B., \& WALKer, J. Modality effects in free recall. Journal of Verbal Learning and Verbal Behavior, $1969,8,665-676$.

NeIsser, U. Cognitive psychology. New York: AppletonCentury-Crofts. 1967.

Smith, E. E., \& SPOEHR, K. T. The perception of printed English: A theoretical perspective. In B. Kantowitz (Ed.), Human information processing: Tutorials in performance and cognition. Potomac, Md: Erlbaum Press, 1974.

Sternberg, S. High-speed scanning in human memory. Science, 1966, 153, 652-654.

STERnBerg, S. Two operations in character recognition: Some evidence from reaction-time measurements. Perception \& Psychophysics, 1967, 2, 45-52.

STERNBERG, S. Memory scanning: Mental processes revealed by reaction-time experiments. American Scientist, 1969, 57, 421-457.

Sternberg, S. Memory scanning: New findings and controversies. Quarterly Journal of Experimental Psychology, 1975, 27, 1-32.

Uhr, L. "Pattern recognition" computers as models for form perception. Psychological Bulletin, 1963, 60, 40-73.

(Received for publication March 31, 1977; revision accepted August 1, 1977.) 